\title{
Enhanced e-Learning Experience using Case based Reasoning Methodology
}

\author{
Swati Shekapure $^{1}$, Dipti D. Patil ${ }^{2}$ \\ Research Scholar, Sant Gadge Baba Amravati University, Amravati, India ${ }^{1}$ \\ Information Technology Department, MKSSS's Cummins College of Engineering for women, Pune, India ${ }^{2}$
}

\begin{abstract}
In recent year's improvement in innovation includes new limits for verifying data that will incite essential changes in eLearning. The user can see e-learning material subject to the reference given to them and select the best approach to see the resources. This proposed system addresses retrieval, reuse, revise and retain phases of CBR. For building personalized e-Learning, this work identifies different feature set such as learning style, learning object, knowledge level, and problem list. For constructing this model used case-based reasoning along with a k-nearest neighbour. Role of the $\mathrm{K}$ nearest neighbour method is to identify the perfect $k$ factor for better analysis for calculation of accurate retrieval process. There is further addition of new cases based on the simulation of new user history limit to a certain threshold value. This model acquires dynamically incremental dataset for classification. Further, there is time and accuracy comparison on dataset done by K-nearest neighbour, decision tree and support vector machine. Eventually, eLearning spares time, upgrades the learning knowledge and gives scholarly achievement.
\end{abstract}

Keywords-K-nearest neighbour method; eLearning; learning objects; learning style; case based reasoning

\section{INTRODUCTION}

The world has changed generously over the most recent 100 years, and instruction needs to change also to guarantee our youngsters are completely arranged Today, a customized learning approach that utilizes innovation in the class room to pace guidance to coordinate student's needs and tailor figuring out how to their interests function for the both students and instructors [4].

Instead of passively accepting and emphasizing data, students' in 21 st century customized learning situations play a functioning job in their training and adds to their very own learning. They can work with instructors to set learning objectives [1] for themselves, and can move in the direction of them through mixed getting the hang of, consolidating face to face collaboration with their educator and the utilization of training innovation.

\section{CASE BASE SySteM}

The term case-based reasoning [8] comprises of three words: case, experience, and problem. A case is an experience of previously occurred problems which are stored in a case base. The representation of cases would do in many ways. A case base is nothing but a collection of represented cases. Store cases are a primary foundation for reasoning. The reasoning to be done in a CBR system is different from an argument in logic and databases. CBR is not based on true rulebooks and accurate decisions. Applying CBR is approximate reasoning. It may happen that the solution in a recorded case was reasonable for its original problem, this would not be the case for a new-fangled problem. This option is created on the universal fact that the condition in the noted knowledge may not be accurate or similar to that in the newfangled problem. The result of the reuse of similar case depends on the similarity of previous experience to a new challenge.

\section{A. CASE}

The evidence documented historical knowledge will be essential, be subject to the area of the inventor, it is called problem space. In the design of a problem-solving a CBR system, the particulars will generally comprise the requirement of the problem and the appropriate characteristics of the situation that are the conditions of the problem. The dynamic part of the case is the explanation that was functional in the previous state. CBR system solution may include facts of the solution or process that are involved in obtaining the solution. It also consists of the attained measure of success in the case explanation, if the cases in the case base have reached different grades of success or failure. When an assessment finished amongst the information stored in a model/rule-based system, and that warehoused in a case base, it seems that the evidence in the case base is of a further detailed nature than that of the model/rule-based system. Although the information in a model/rule-based system has preoccupied so that it related in the broadest diversity of circumstances as possible, the information controlled in case base residues precise to the case in which it is stored. [6] Since the accurate information of a case base, it has been discovered that associated information and knowledge relevant in a particular condition warehoused in neighboring contiguity. Therefore, relatively illustrating information from an extensive net, the information desired to answer a precise unruly instance can establish a cluster. The case base in the CBR structure is the recollection of all earlier warehoused cases [14]. Three broad areas have to consider when creating a case base.

- The construction and illustration of the instances themselves.

- The recollection prototype used for establishing the entire case base.

- The choice of keys which are used to categorize every case. 


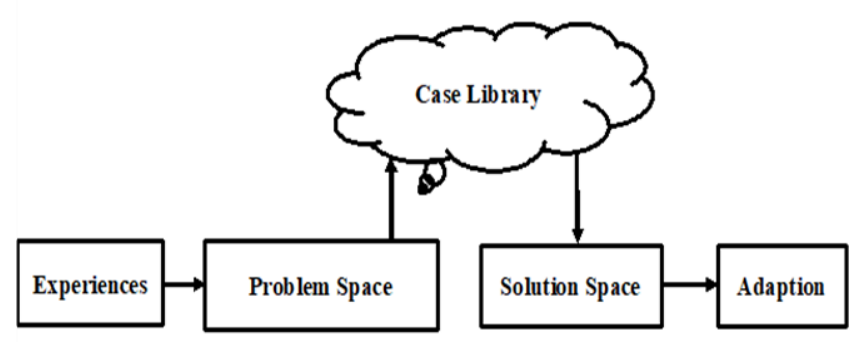

Fig. 1. Solution Space of CBR.

\section{B. Experiences}

Experiences are vital for the CBR system. Experiences have documented an event that happened in the prior. Cases can be relatively composite and comprise of entire stories. CBR practices them for answering problems, and these must be in the knowledge base for talks about a problem and its solution. In a simple view, CBR splits a piece of knowledge into two parts shown in Fig. 1.

a) A problem space (or an explanation of a problem situation).

b) A solution space that defines in what way one has responded.

Over and over again one limits CBR to a solution that has been positively, but that is by no means essential or satisfactory. An unsuccessful explanation is also a significant part of the evidence that states what one has to circumvent. Positive experiences implement practical solutions and lead to guidance. Negative experiences implement unsuccessful solutions and lead to advise that avoids this case.

Essential experiences take place in:

a) Classification: Select the class to which an entity be appropriate.

b) Diagnosis: Select what the analysis of a problem is.

c) Prediction: Answered what happens in the future.

d) Planning: select an order of activities to extend a given goal.

e) Configuration: Select technical features to include.

Although a human can learn through experiences, for computer system experiences are represented in the form of cases and stored in the case library. A new incident occurred then the solution space would retrieve a given solution for adaption.

\section{Problems and Solution}

The primary purpose of CBR is to solve the problems. The preparation of a challenge from time to time is challenging because it refers to the situation in which it has been specified. So, each problem development involves not the same kind of explanation. It is understandable that one has to identify the condition in which the problem has been specified to discover which answer is suitable. In further, for a detailed report, the situation has to be involved in the problem preparation. Fragment of the case is repeatedly of the fundamental principles. For example, Depending on the values, rules may have changed in different spaces. Other beliefs provided by diversified fields such as medicine, commercial, industrial, and engineering; even outsized corporations have established their principles. The CBR background has to consider this into interpretation because transmitting justifications across different cultures is challenging. For illustration, every bank has advanced its strategy for giving credits to clients. The same bank possibly will interpret the policy in a different way in each separate nation it activates; this becomes superficial during economic crunches. There are two types of problems in the framework of the CBR method. The issues in the cases verified as familiarities usually denoted as problems in CBR. The facts in the case base can distinguish as contender cases, as they are contenders for reprocess. However, the entire CBR progression has activated by a problem. A new challenge or the concrete problem encourages a user to discover a problemsolving technique [10].

The probable ways of demonstrating an explanation differ: It can be just an explanation in the contracted sense. It can comprise notes, graphics, clarifications and guidance on how to use the statement. The consequence by telling what happened with the solution in the earlier. Comments on the approach with which, the clarification has been obtained. In modest cases, the description comprises a name or small data, for example, an object or an estimated high temperature. Explanations may also have a complex object-oriented configuration as a methodological object. Even more composite solutions for scheduling and those in documentary or image form. In a composite situation, the answer is a conclusion for acting or even a process.

Here one has to differentiate the outcome from the action; the action mentions to execute and run of a plan that may alter states of variables. Whereas the conclusion generally communicated, the result of the work may be indeterminate. Suppose for instance if it has been decided to fly to Mumbai. The execution may fail or be suspended because of numerous unexpected happenings. The latter means that the outcome of using a solution is ambiguous because of unforeseen exterior consequences like bad weather or an earthquake. If these are expected to occur one should extend the explanation by an entry "consequence" for telling what happened. The user who understands the statement does not recognize this. If it is added at that moment, the user might get a clue for specific possible reworking. In conclusion, there are circumstances where the effectiveness of the explanations can only judge if they have implemented in reality [12].

\section{NEIGHBOURHOOD FORMATION PROCESS}

Proposed work deals into various assignments, for example, a. make a database with the solicitation to perceive learning style b. Adding learning Material and essential KNN for the recommendation c. Adding Test Question and Revise Retain Algorithm execution d. Checking Comparisons with other ML estimations. This is an adaptable recommendation structure for endorsing learning preferences to the user dependent on CBR and seeing the closest neighbour. This work proposed to fulfil four phases of CBR these are retrieve, reuse, revise and retain as appeared in Fig. 2. It gets understudy's tendencies [2] by finishing express fundamental 
outlines, ensuring to examine recommend material. User can use certain learning objects for understanding a specific point. For examining self-execution user attempt the test. Whatever execution of user gets contrasted and the limit esteem. On the off chance that it fulfilling limits, at that point it gets put away for the case library. This work uses case library data as the strategy set. Right when another issue exists proposed structure searches for after case-based reasoning impels. It has point by point differentiating cases, and errand of referencing had done, when the new case exists to get empowered by considering closeness vertex and find the nearest case interfacing with another case.

For identifying new user behavior [11], it has conspired different feature set; these are:

\section{Feature set for the system}

1. Problem List $=$ ffor, if, if else, while, do while, switch

2. Learning Styles $=\{$ Visual, Auditory, Kinaesthetic $\}$

3. Knowledge Levels $=\{$ Beginner, Intermediate, Expert\}

4. Learning Objects $=\{$ Video, Chart, Audios, Simulation, Highlighted Text $\}$

5. Test Performance $=[3,4,5,6,7]$

6. path $=\{$ Video- $>$ Chart, Audios- $>$ Video, Simulation$>$ Highlighted Text

Related values of input parameters are in Table I.

After conspicuous verification of feature vectors and association between things, the accompanying stage is to make cases for rundown out of abilities by considering historical results. This work has framed diverse cases with id, problem, learning style [9], learning objects, test execution and utilized way. This model had considered preparing a dataset for a finding of proper estimation of $\mathrm{K}$. When the finding of estimation of $\mathrm{K}$ had done then $\mathrm{KNN}$ is prepared for producing similitude list for new user. On the off chance that the new user didn't get the comparative case, at that point, this work checked new client premium, whatever way embraced for learning and test execution score. In the event that scores gotten by a new user is acceptable according to edge esteem, at that point the particular case gets put away in database with ordering.

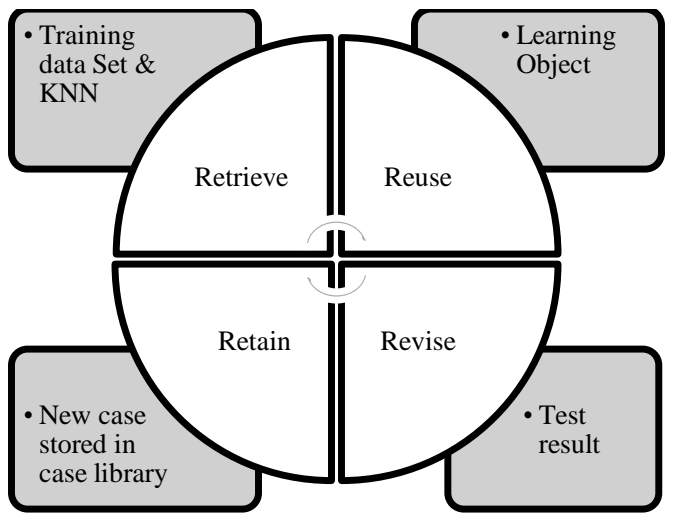

Fig. 2. CBR Phases.
TABLE I. Feature Set VAlues

\begin{tabular}{|c|c|c|c|}
\hline $\begin{array}{l}\text { Sr. } \\
\text { No. }\end{array}$ & Input parameter & Feature set & Value \\
\hline \multirow{6}{*}{1} & \multirow{6}{*}{ Problem } & For & 1 \\
\hline & & If & 2 \\
\hline & & If-else & 3 \\
\hline & & while & 4 \\
\hline & & Do while & 5 \\
\hline & & switch & 6 \\
\hline \multirow{3}{*}{2} & \multirow{3}{*}{ Learning Style } & Visual & 1 \\
\hline & & Auditory & 2 \\
\hline & & Kinaesthetic & 3 \\
\hline \multirow{3}{*}{3} & \multirow{3}{*}{ Knowledge level } & Beginner & 1 \\
\hline & & Intermediate & 2 \\
\hline & & Expert & 3 \\
\hline \multirow{5}{*}{4} & \multirow{5}{*}{ Learning object } & Video & 1 \\
\hline & & Chart & 2 \\
\hline & & Audios & 3 \\
\hline & & Simulation & 4 \\
\hline & & Highlighted text & 5 \\
\hline 5 & Test Performance & Result & Actual Result \\
\hline \multirow{3}{*}{6} & \multirow{3}{*}{ Path } & Video-chart & 1 \\
\hline & & Audios-video & 2 \\
\hline & & $\begin{array}{l}\text { Simulation-Highlighted } \\
\text { Text }\end{array}$ & 3 \\
\hline
\end{tabular}

IV. REVIS-RETAIN ALGORITHM IMPLEMENTATION

Revise and Retain phase of case based reasoning is to add new query with new index value in dataset. This is dynamically incremental phase of CBR whereas assignment of new index value had done by considering test performance of new user. This has finished by simulating result and threshold value set [3]. This phase compromises different work through here presented few algorithms for revise and retain phase implementation. For that initially load training and testing dataset so algorithm represents fetching of input parameters. After loading dataset as training and testing, next step is to check similarity .This research work emphasis on CBR with KNN .So for finding similar case Euclidian distance measures had used. Below presenting how Euclidian distance measure works on input dataset for calculating class of new user. After calculating values of learning style, knowledge level, and test performance the job of retain and revise phase suggest to learning path to new user. So whatever score has established for $\mathrm{x}$ value dataset need to normalized obtained weight.

For normalizing input set practice equation 1 :

$Z i=\frac{x i-\min (x)}{\max (x)-\min (x)}$

Where $\mathrm{x}=\left(\mathrm{x}_{1} \ldots \mathrm{x}_{\mathrm{n}}\right), \mathrm{Zi}$ is $\mathrm{i}^{\text {th }}$ normalized data. 
Algorithm for Proposed System:

1) Identify training set and test set as well as attribute for prediction

i) Load Dataset (trainingset, testset, attr)

ii) attr is "Learning Objects"

2) For each test element in test set calculate neighbours

i) getNeighbors(traningSet, testset[x], $\mathrm{k}$, attr)

ii) Parameters: training Set, single test element $\mathrm{x}, \mathrm{k}$, attr= "Learning Object"

3) In getNeighbours calculate score one by one with each training element

i) For $\mathrm{x}$ in traningSet

ii) getScore(x,testInstance,attr)

iii) $\mathrm{x}=$ training element, test element, attr= "LearningObject"

4) In getScore calculate score for each feature in element

i) getScore(x,testInstance,attr)

ii) LearningStyleScore $=$ calculate (x.learningStyle,testInstance.learningStyle, data.le arningstyle)

iii) ProblemScore $=$ calculate (x.problem,testInstance.problem,data.problem)

iv) KnowledgelevelScore $=$ calculate (x.Klevel,testInstance.Klevel,data.Klevel)

v) PathScore $=$ calculate $($ x.path,testInstance.path,data .path)

5) In calculate

i) Calculate(xValue,testValue,dictVals)

ii) For calculating Learningstyle, $\mathrm{x}$ Value $=$ LearningStyle for traning element (e.g. Visual),

iii) testValue(e.g.Visual),

iv) $\operatorname{dictVals}=\{$ "Visual $=1 "$, "Auditory=2","Kinesthetic=3"

6) As per $\mathrm{KNN}$ for each feature distance $=$ square(trainingValue - testValue)

i) $\operatorname{Pow}(\mathrm{v} 1, \mathrm{v} 2)^{2}$

7) As per KNN, Total distance $=$ squareRoot $($ total $)$

8) Sort all the elements in training set based on distance, as $\mathrm{k}=4$ select first 4 nearest elements

i) Neighbours $=$ []

ii) For $\mathrm{x}$ in sorted (distances)

iii) If $(\mathrm{k}==0)$

iv) Break;

v) Neighbours. Append (distances[x])

vi) $\mathrm{K}=\mathrm{k}-1$;

vii) Return neighbours.

9) After getting neighbours, predict feature

i) Result=getResponse(neighbors,attr)

ii) Attr= "learningObject"

10) In get Response, this work find attr=LearningObject value count

i) For example

ii) first neighbour value $=$ Visual

iii) second neighbour value=visual

iv) third neighbour value $=$ Visual

v) fourth neighbour value=Auditory

vi) then,

vii) classVotes $=\{$ Visual $=3$, Auditory $=1\}$

viii) Then sorting of above map, to get highest voted value, here "Visual"

11) Next step is to check this value with test instance and calculate accuracy

\section{RESULT}

As a part of result, proposed framework classes' students according to the learning style [7] as visual, Auditory and Kinesthetic. Result introduced for learning style just as the recommendation of learning object to another student. Alongside recommendation, there is learning material benefited to a student. Learning material is as a diagram, graph, notes, simulation, and featured content. Fig. 3 demonstrates identification of learning style of the user.

According to learning style, problem and knowledge level of student (Fig. 4) system shows recommendation to learner as shown in Fig. 5.

$\rightarrow$ c localhost:5000/questions
1. What I generally do while operating new equipment
A. First I read the instructions
B. I take note of an description given by somebody who has used it before
C. I will go ahead and operate it, and figure out after using that equipment
2. Which guideline is better for me related to directions while travelling
A. I will use a map
B. I will ask to nearby person for directions

Fig. 3. Questionnaire's Set for Learning Style.

\begin{tabular}{|l|}
\hline (i) localhost $5000 /$ post \\
You have Visual Learning Style \\
Which control loop you want to learn? \\
1. For \\
2. If \\
3. If else \\
4. While \\
5. Do While \\
6. Switch
\end{tabular}

Fig. 4. Generated Learning Style and Input Problem.

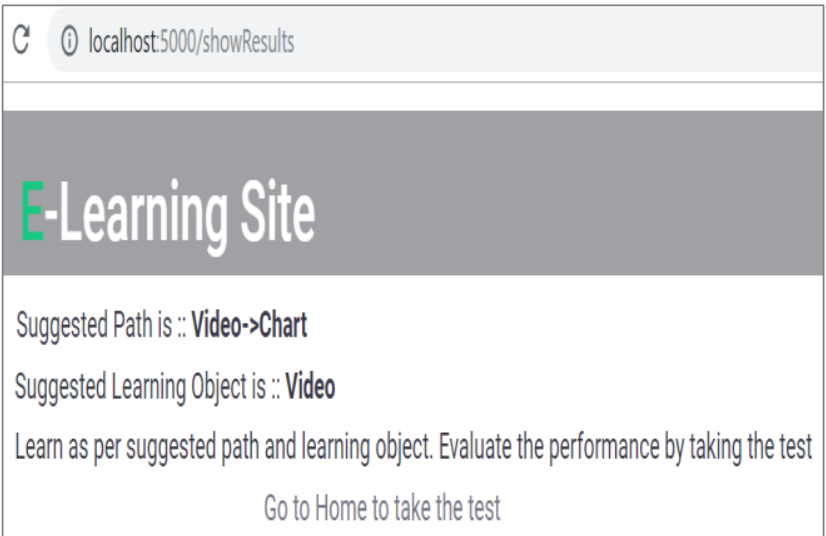

Fig. 5. Recommendation of Learning Objects and Path. 
Fig. 6 shows sample set for test performance. The sets depend on input received from user whether learner is beginner, Intermediate or expert in that particular topic. After using different learning objects, student opts for the test.

If the test performance is satisfied then add student path includes learning objects and test performance to a dataset of case based reasoning. Satisfactory performance of student indicates that simulation done in real-world scenario and verified by domain experts. It has retained phase of CBR.

Fig. 7 shows retain phase of CBR

For retrieval phase, similarity check is done on different classification algorithm. These are a k-nearest neighbor, decision tree and support vector machine. For this research work, consideration of dataset size is 200 records, 300 records and 375 records. Table II shows observations for 375 records.

It has been observed that for 200 records decision tree has given results in least amount of time as compared to k-nearest neighbor method and support vector machine.

Fig. 8 shows that average time observations of different learning algorithms. It has been observed that Decision tree takes less time as compared to KNN and SVM, but KNN gives retrieval phase stability which is more as compares to DT and SVM. [15].

Further this work calculated accuracy of decision prediction by above learning algorithm. Fig. 9 shows the observations for 200 records size, 300 records size and 375 record size.

As per the observation it has been found that $\mathrm{KNN}$ and decision tree gives maximum accuracy as compared to SVM. But for time comparison KNN timing for retrieval process is stable as compared to Decision tree and Support vector machine. So for this dataset it has better to use KNN than DT and SVM.

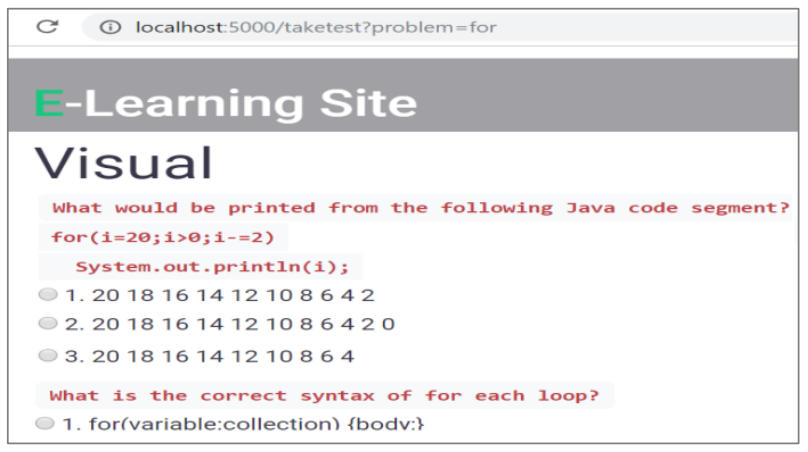

Fig. 6. Set for Test Performance.

\begin{tabular}{|l|}
\hline C (1) localhost:5000/showResults1 \\
\hline E-Learning Site \\
Marks :: 10 \\
Great. You passed the test. We have Retained the data to dataset \\
\hline
\end{tabular}

Fig. 7. Retain Phase of CBR.
TABLE II. OBSERVATIONS FOR 375 RECORDS, TIME IN MILLISECONDS

\begin{tabular}{|c|c|c|c|}
\hline Sr. No. & KNN & SVM & DT \\
\hline 1 & 5.23 & 10 & 5 \\
\hline 2 & 5.8 & 10 & 6 \\
\hline 3 & 7.6 & 10 & 5 \\
\hline 4 & 5.6 & 8 & 4 \\
\hline 5 & 5.76 & 7 & 4 \\
\hline 6 & 5.1 & 6 & 4 \\
\hline 7 & 5.23 & 8 & 6 \\
\hline 8 & 5.53 & 7 & 4 \\
\hline 9 & 4.8 & 7 & 4 \\
\hline 10 & 5.53 & 6 & 4 \\
\hline 11 & 4.53 & 7 & 4 \\
\hline 12 & 5.06 & 8 & 7 \\
\hline 13 & 5.2 & 7 & 4 \\
\hline 14 & 4.7 & 7 & 5 \\
\hline 15 & 4.86 & 7 & 4 \\
\hline 16 & 8.1 & 10 & 6 \\
\hline 17 & 6.1 & 7 & 4 \\
\hline 18 & 4.9 & 8 & 4 \\
\hline 19 & 5.46 & 7 & 5 \\
\hline 20 & 4.83 & 7 & 4 \\
\hline Average & $5.49 \mathrm{~ms}$ & $7.7 \mathrm{~ms}$ & $4.65 \mathrm{~m}$ \\
\hline
\end{tabular}

\section{Average Time Comparison}

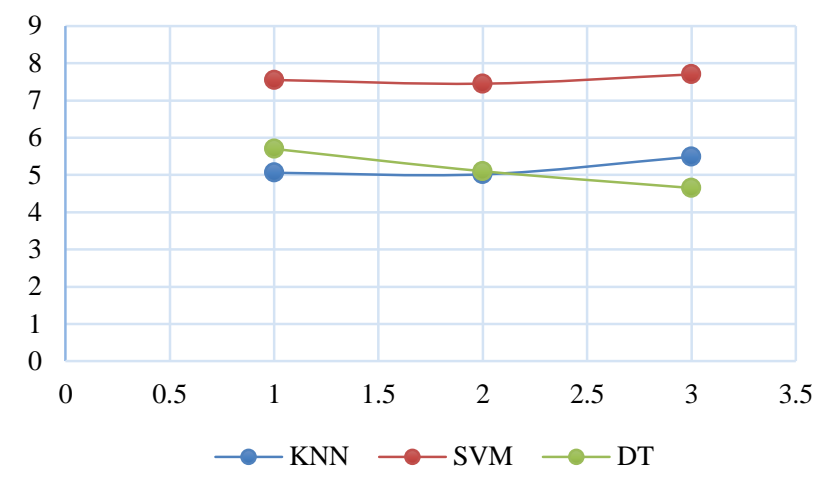

Fig. 8. Average Time Comparison of KNN, SVM and DT.

\section{Accuracy Comparison}

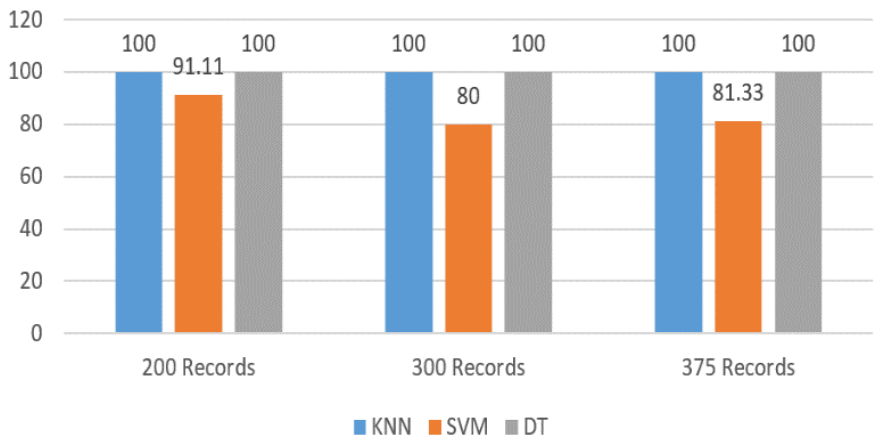

Fig. 9. Accuracy Comparison of KNN, SVM and DT. 


\section{CONCLUSION}

This research paper focuses on the current technology of internet e-learning. This methodology is appropriate for applications in e-learning such as to enhance the website to the personalized facility. Generation of the weblog is dynamic activity so each time our approach generates unambiguous results, that is, key behind this research work to focus on learner's interest as well as their performance. This is Adaptive personalized e-learning system [10] because whatever path used by learner's for specific topic get stored in dataset with new index value. So new user able to check different learning objects and impact of it on performance. Because of this learners way of learning and time automatically hold back for understanding theories. It will definitely be beneficial for younger generations particularly teenagers where they are in position to acquire knowledge[13] in least time with more understanding approach.

In future, the learning solutions and services can be integrated into a new trend which is called mobile learning technology [5] that can help the people who are lack of infrastructure and for the people whose job require to move.

\section{DISCUSSION}

In view of student's education to develop students' learning interest and improve performance, a case based personalized eLearning system must know how a different learner learns best. In this innovative framework, work presented for learners modelling through dynamically incremental model of case library. Research have exposed this methodology is able to recommends learning objects , automatically records the path and adjust learner's learning style, based on k-nearest neighbor method for retrieval similar interest.

\section{ACKNOWLEDGMENT}

It's with immense gratitude that we acknowledge Dr. V.M. Thakare, Head P. G. Department of Computer Sci. \& Technology, Sant Gadge Baba Amravati University, Amravati for sharing his wide knowledge, co-operation from time to time and prestigious guidance during the preparation of this work.

\section{REFERENCES}

[1] Kwok Hung Lau, Tri-Lam, Booi HonKam, Mathews Nkhoma, Joan Richardson, Susan Thomas, " The role of textbook learning resources in e-learning: A taxonomic study, Computers \& Education, Elsevier,Volume 118, pp 10-24,March 2018.

[2] Beulah Christalin LathaChristudas, E.Kirubakaran, P. Ranjit JebaThangaiah, "An evolutionary approach for personalization of content delivery in e-learning systems based on learner behavior forcing compatibility of learning materials," Telematics and Informatics, Elsevier, Volume 35, Issue 3, pp. 520-533, June 2018.

[3] Aleksandra Klasnja-Milicevic, Mirjana Ivanovic, Boban Vesin, Zoran Budimac, "Enhancing e-learning systems with a personalized recommendation based on collaborative tagging techniques," Applied Intelligence, Springer Volume 48, Issue 6, pp. 1519- 1535,2018.

[4] Wacharawan Intayoad, Till Becker, Punnarumol Temdee, "Social Context-Aware recommendation for Personalized Online Learning," Wireless Personal Communications, Springer ,Volume 97, pp. 163179, November 2017.

[5] Peter Dolog, Michael Sintek, "Personalization in Distributed e-Learning Environments," May 17-22, 2017, New York, USA. ACM 1-58113912-8/04/0005

[6] Khribi, M. K., Jemni, M., \& Nasraoui, O. . ." Automatic Recommendations for E-Learning Personalization", Based on Web Usage Mining Techniques and Information Retrieval. Educational Technology \& Society, IEEE, pp. 30-42,2017.

[7] Kusuma Ayu Laksitowening; Amarilis Putri Yanuarifiani; Yanuar Firdaus Arie Wibowo, "Enhancing e-learning system to support learning style based personalization," IEEE,16 February 2017.

[8] Antonio Garrido, Lluvia Morales, Ivan Serina, "On the use of casebased planning for learning personalization," Science Direct, 30 October 2016, Pages 1-15.

[9] Birol Ciloglugil, Mustafa Murat Inceoglu, "A Felder and Silverman Learning Styles ModelBased Personalization Approach to Recommend Learning Objects," ,Springer,01 July 2016.

[10] Boban Vesin, Mirjana Ivanović, Zoran Budimac, Lakhmi C. Jain, "Personalization and Adaptation in E-Learning Systems," Springer,20 July 2016.

[11] Beulah Christalin, Latha Christudasa, E.Karunakaran, P. Ranjit JebaThangaiaha, "An evolutionary approach for personalization of content delivery in e-learning systems based on learner behavior forcing compatibility of learning materials," Elsevier,4 Feb 2017.

[12] Outmane Bourkoukou, Essaid El Bachari, and Mohamed El Adnani, "A Personalized eLearning Based on Recommender System," International Journal of Learning and Teaching, 2016.

[13] Fatemeh Roosta; Fattaneh Taghiyareh; Maedeh Mosharraf, "Personalization of gamification-elements in an e-learning environment based on learners' motivation," IEEE, 20 March 2017.

[14] Shard from Johnson; Xubin Liu; Hui Miao; Jiajin Yuan; Yong Jin; Qin Wei; Zhao Xu, "A Framework of e-Learning Education Clouds to Efficiency and Personalization," IEEE, 3 November 2016.

[15] Elfa Silfiana Amir; Malikus Sumadyo; Dana Indra Sensuse; Yudho Giri Sucahyo; Harry Budi Santoso, "Automatic detection of learning styles in learning management system by using literature-based method and support vector machine," IEEE, 09 March 2017.

\section{ABBREVIATIONS}

1. KNN: K-Nearest Neighbour

2. DT: Decision Tree

3. SVM: Support Vector Method

4. CBR: Case Based Reasoning 